\title{
Simultaneous Linear Recurrence Relations with Variable Coefficients
}

\author{
By H. D. Urgell \\ (Received 11th November, 1955. Revised 14th February, 1957.) \\ 1. Introduction.
}

Our subject ${ }^{1}$ is a set of equations

$$
\sum_{j} A_{i j}(n) u_{j}(n)=z_{i}(n)
$$

where the $u,(n)(j=1,2, \ldots, k)$ are $k$ " unknown " functions of the integer variable $n$, the $z_{i}(n)(i=1,2, \ldots, h)$ are $h$ " known" functions of $n$, and the $A_{i j}(n)$ are $h k$ "known" operators

$$
A_{i j}(n)=\sum_{(v)} \alpha_{i j}^{(v)}(n) E^{\nu}
$$

which are polynomials in $E$, each of fixed order $p_{i j}$ but with coefficients which may vary with $n . \quad E$ is the usual operator defined by

$$
E f(n)=f(n+1) .
$$

Our first task is to determine whether the equations (1) are selfconsistent. Secondly, if they are self-consistent, we ask what follows from them for a given subset of the unknowns, e.g. for $\left(u_{j+1}, \ldots, u_{k}\right)$, in other words we wish to eliminate $\left(u_{1}, \ldots, u_{j}\right)$. In particular we wish to

1 We shall keep to this application. But, as a referee has noted, much of our work applies to any elements $A_{i j}$ belonging to a (non-commutative) ring which possesses a right (and for some purposes a left) Euclidean algorism : in particular it applies to differential operators.

In the purely algebraic context, we would draw attention to the definition of the row-rank $\rho$ (column-rank $\kappa$ ) of a matrix when the ring possesses a right (left) algorism, to the reduction to $\rho$ rows ( $\kappa$ columns), and to the equality of $\rho$ and $\kappa$ when both algorisms are available.

In the context of differential equations, the H.C.R.F. process appears to be known, but not well-known. The order of a system " in general " (and therefore also of the eliminant " in general") is given already by Jacobi (Crelle, 64, 297; Werke, V, 193) as $p$ of our $(45 \cdot 1)$, §6. There is no apparent analogue in this case of the $\epsilon$ of our $(45 \cdot 2)$. Jacobi states the result for non-linear simultaneous differential equations. His first two steps, reducing the problem to one of homogeneous linear equations with constant coefficients, seem to the writer not entirely satisfying in these days : that he finds no need of such considerations as are involved in our two lemmas arises partly from this, partly from the fact that he concerns himself with the order of the system, not that of the eliminant. 
eliminate all the variables but one, say $u_{k}$. We shall in fact find that either $u_{k}$ is arbitrary or else that it has only to satisfy a single linear recurrence relation : and the order of that relation is of interest to us. Thirdly, we ask that reduction to standard form is possible, with or without a transformation of the unknowns themselves.

In the case of homogeneous equations

$$
\sum_{j} A_{i j} u_{j}=0
$$

the problem of self-consistency is altered. There is the obvious trivial solution

$$
u_{j}=0 \quad(\text { all } n, j)
$$

and so we ask whether any other solution exists. But this is not the whole story, as we see by considering the single equation in one unknown

$$
E^{3} u=0 \text {. }
$$

If we regard $n$ as running through all integers, positive, negative and zero, this equation has only the trivial solution $u=0$. But if, as is more usual in connection with recurrence relations, $n$ is to take only nonnegative or positive values, then the equation has the additional solution given by $u(1)=1, u(n)=0$ for $n \neq 1$. It will be convenient to lump together as "trivial" all solutions for which

$$
u_{j}=0 \quad(\text { all } j \text {, all large } n) .
$$

It will be observed that we have written $A_{i j}, u_{j}$ instead of $A_{i j}(n), u_{j}(n)$. In order to simplify the notation we shall usually suppress any explicit mention of $n$ : but before $n$ is forgotten entirely, two remarks must be made.

Firstly: the manipulations we describe produce at every stage new quantities which, like the old, are functions of $n$. The march of our manipulations depends on whether these functions of $n$ "vanish" or not. To "vanish" will mean to vanish identically in $n$ : but difficulty arises if one of these quantities, while not vanishing identically, does vanish for a particular value of $n$. Our work has little value if these " particular" values of $n$ are more than finite in number.

Secondly: if the operators $A_{i j}$ were independent of $n$, our problems would be solved by classical procedures : thus the whole point of our work lies in the fact that it still applies with variable operators $A_{i j}$. In our case some of the classical methods fail-among them the most powerful 
method of the eigen-values of $E$-and we must pick our way rather carefully even to get a linear recurrence relation for $u_{k}$.

The process of elimination from simultaneous recurrence relations leads us, as a by-product, to the (non-linear) recurrence relation satisfied by the convergents of a continued fraction. It seems unlikely that this relation is new, but it is certainly little known.

We are also led to define the rank of a matrix of operators of the type (2). Here again care is needed: we have no analogue of the subdeterminants that figure in any classical treatment of the rank of a matrix.

This note was prompted by the paper "A Determinantal Expansion for a Class of Definite Integral, Part 5", by L. R. Shenton, Proc. Edinburgh Math Soc. (2), 10 (1957), 167-188 (169), which the present writer was privileged to see before its publication.

\section{Simultaneous Equations in One Unknown and the H.C.R.F. of a Set of Operators.}

Consider the $h$ simultaneous equations

$$
A_{i}(n) u(n)=\zeta_{i}(n)
$$

in one unknown $u(n)$. The $A_{i}$ are polynomials in $E$. We adopt a process corresponding to that for constructing the H.C.F. (highest common factor) of $h$ polynomials in an ordinary variable. We select one of them (say $A_{\beta}$ ) whose order in $E$ is least. We divide the other $A_{i}$ on the right by $A_{\beta}$ obtaining equations

$$
A_{i}=t_{i} A_{\beta}+A_{i}^{\prime}
$$

where $t_{i}, A_{i}{ }^{\prime}$ are new polynomial operators and $A_{i}{ }^{\prime}$ is of lower order than $A_{\beta}$. We also put

$$
A^{\prime}=A_{\beta}
$$

The change from the set $\left\{A_{i}\right\}$ of operators, written as a column, to the column $\left\{A_{i}^{\prime}\right\}$ may be represented by

$$
\left\{A_{i}\right\}=t .\left\{A_{i}{ }^{\prime}\right\}
$$

where $t$ is a matrix operator having unity in each element on the principal diagonal, $t_{i}$ in the $(i, \beta)$ place, and zero elsewhere. We have also

$$
\left\{A_{i}{ }^{\prime}\right\}=s \cdot\left\{A_{i}\right\}
$$

where $s=2 I-t, I$ being the unit matrix. $s, t$ are inverse operators:

$$
s t=t s=I \text {. }
$$


We apply the operator-matrix $s$ on the left to the column of equations (7), obtaining

$$
A_{i}{ }^{\prime} u=s\left\{A_{i}\right\} u=s\left\{\zeta_{i}\right\}=\zeta_{i}^{\prime},
$$

say. The new set of equations is completely equivalent to the old, since the old may be recovered by applying the operator matrix $t$ to the new.

This process of reduction is now repeated as many times as possible. At each step, except possibly the first, the highest order of the operators $A_{i}$ is lowered: hence the process terminates after a finite number of steps. When it terminates all the operators $A_{i}$ but one have been reduced to zero.

Let $s^{(1)}, t^{(1)} ; \ldots ; s^{(p)}, t^{(p)}$ be the pairs of transforming matrices used in the different steps and write

Then clearly

$$
S=s^{(p)} \ldots s^{(1)}, \quad T=t^{(1)} \ldots t^{(p)} .
$$

$$
S T=T S=I .
$$

We may suppose

$$
S\left(\begin{array}{c}
A_{1} \\
\vdots \\
A_{h}
\end{array}\right)=\left(\begin{array}{c}
F \\
0 \\
\vdots \\
0
\end{array}\right), \quad T\left(\begin{array}{c}
F \\
0 \\
\vdots \\
0
\end{array}\right)=\left(\begin{array}{c}
A_{1} \\
\vdots \\
A_{h}
\end{array}\right),
$$

since the one non-zero element surviving may be brought into this position by one more matrix multiplication. Then

$$
A_{i}=T_{i 1} F, \quad F=\sum_{i} S_{1 i} A_{i} .
$$

The equations (17) justify calling $F$ the highest common right factor (H.C.R.F.) of the $A_{i}$ [the elements of $S, T$ are by (14) polynomials in $E$ ] and the H.C.R.F. is unique save for an arbitrary left factor not involving $\boldsymbol{E}$.

By an operator we shall mean always a polynomial in $E$, and by an operator matrix (or matrix operator) we shall mean a matrix whose elements are operators. A (square) matrix operator will be called regular if it has an inverse: thus $S, T$ are regular, and the product of two regular matrices is regular. Multiplying a set of equations on the left by a regular matrix operator gives a new set of equations completely equivalent to the old. And by such transformation we can reduce the equations (7) to the form

$$
\begin{gathered}
F u=Z_{1}, \\
0=Z_{i} \quad(i>1),
\end{gathered}
$$

where $Z_{i}=S\left\{\zeta_{i}\right\}$. 
This may be considered as solving the problem of the elimination of one unknown from a system of linear recurrence equations. The equations (18.2) are the result of elimination, and if they are satisfied then the sole condition on $u$ is the single linear recurrence relation (18.1).

To reduce the equations to the form (18), however, we must know the operators $A_{i}$. The $\zeta_{i}$ may, for example, contain certain parameters, and then (18.2) gives the conditions on these parameters in order that (7) be self-consistent: but we cannot effectively reach (18) unless the $A_{i}$ are genuinely known. If we try to follow out the process of reduction without that knowledge, we are led to a string of alternatives of the type of (18) which apply according as certain combinations of the coefficients in the operators $A_{i}$ do or do not vanish.

We may ask whether by some other method we can carry out the elimination and obtain a single set of equations which will include all these alternatives. The answer appears to be in the negative unless we are prepared to accept non-algebraic equations. For example, the result of eliminating $u$ from the simultaneous equations

$$
(a E+b) u=0, \quad\left(a^{\prime} E+b^{\prime}\right) u=0
$$

is " in general"

$$
a b^{\prime}-a^{\prime} b=0 .
$$

But if $a=a^{\prime}=0$, and $b=b^{\prime}=1$, this condition is satisfied although the equations for $u$ have no common non-trivial solution. The true result of the elimination gives all points (in the space of coordinates $a, b, a^{\prime}, b^{\prime}$ ) for which $a b^{\prime}=a^{\prime} b$, excluding those for which $a=a^{\prime}=0$, excluding also those for which $b=b^{\prime}=0$, but including the point $a=a^{\prime}=b=b^{\prime}=0$. This construct, which as a set of points is not closed, cannot be given by algebraic equations.

To summarise: the $h$ equations (7) in one "unknown" can be reduced, by operating on the left with a regular matrix operator, to the single equation (18.1) for the unknown and the $(h-1)$ equations (18.2) free of the unknown. The operator $F$ is the H.C.R.F. of the $A_{i}$ and does not vanish (identically) unless all the $A_{i}$ vanish (identically). This exception, which can arise non-trivially in our later work, can be included formally as a case in which $F=0$ : from the point of view of elimination, of course, (18.1) disappears and all $h$ equations appear in (18.2).

\section{The Rank of a Matrix Operator.}

Suppose that we are given an $(h, k)$ matrix operator $A=\left\|A_{i j}\right\|$. We shall first discuss the dependence or independence of the rows of $A$, and 
the forms to which it can be reduced by multiplication on the left by a regular matrix operator. The description of the results is simplified if we allow ourselves on occasion to transpose columns: such transposition is equivalent to multiplying the matrix on the right by a regular matrix (of constants), but the role of these right factors is quite subsidiary.

If $A$ does not vanish identically, we choose a column which does not vanish identically (e.g. the first such column) and transpose this column to the first position. This transposition is given by a matrix $C^{(\mathbf{1})}: A C^{(\mathbf{1})}$ has first column not identically zero.

Next we choose the regular matrix operator $S^{(1)}$ so that $S^{(1)} A C^{(1)}$ has a first column

$$
\left(\begin{array}{c}
F_{1} \\
0 \\
\vdots \\
0
\end{array}\right) \text {. }
$$

From now on we are concerned only with the last $(h-1)$ rows and last $(k-1)$ columns of $S^{(1)} A C^{(1)}$. Our programme is completed if either $h=1$ or $k=1$ or the submatrix formed by the common part of these rows and these columns vanishes identically. If not, we choose a column of the submatrix which does not vanish identically, bring it to the first position in the submatrix, i.e. to the second position in the whole matrix, by a transposition $C^{(2)}$, leaving our first column, of course, in place. By a regular matrix operator $S^{(2)}$ which leaves the first row unchanged and only transforms the remaining $(k-1)$ rows among themselves we bring the first column of the submatrix to standard form. Continuing in this way we reach our end after, say, $r$ steps. Writing

$$
S=S^{(r)} \ldots S^{(1)}, \quad C=C^{(1)} \ldots C^{(r)},
$$

we see that $T$ is a regular matrix operator, that $C$ merely permutes the columns in some way, and that

$$
S A C=\left(\begin{array}{ccccc}
F_{1} & \ldots & \ldots & \ldots & \ldots \\
& F_{2} & \ldots & \ldots & \ldots \\
& & \ddots & \ldots & \ldots \\
& & & F_{r} & \ldots
\end{array}\right)
$$

where all elements below either the principal diagonal or the $r$-th row vanish, while $F_{1}, F_{2}, \ldots, F_{r}$ are in fact different from zero.

Let us say that $\eta$ rows $R_{1}, \ldots, R_{\eta}$ of a matrix operator $M$ are dependent 
if there exists a row $B=\left(B_{1}, \ldots, B_{\eta}\right)$ of operators not all zero and such that

i.e.

$$
\begin{gathered}
B_{1} R_{1}+\ldots+B_{\eta} R=0, \\
B_{1} R_{1 j}+\ldots+B_{\eta} R_{\eta j}=0 \quad(\text { all } j),
\end{gathered}
$$

where $R_{1 j}, \ldots, R_{\eta j}$ are the $j$-th elements of $R_{1}, \ldots, R_{\eta}$. If no such $B$ exists we say that the rows $R_{1}, \ldots, R_{\eta}$ are independent. Let us also say that $\rho$ is the row-rank of a matrix $M$ if there is in $M$ at least one set of $\rho$ rows which are independent, while any $(\rho+1)$ rows of $M$ are dependent.

It is evident that $r$ is the row-rank of the matrix $S A C$ in (20), and that multiplication on the right by a transposition matrix $C$, or even by any regular matrix, cannot alter the row-rank of a matrix.

Now consider a matrix $M$ of row-rank $\rho$. We suppose without real loss of generality that the first $\rho$ rows $R_{1}, \ldots, R_{\rho}$ of $M$ are independent. There is a relation

$$
B_{1} R_{1}+\ldots+B_{\rho} R_{\rho}+B_{\rho+1} R_{\rho+1}=0
$$

between the first $(\rho+1)$ rows. The process of forming the highest common left factor $G$ of the $B_{i}$ leads us to a regular matrix $t$ (of $\rho+1$ rows and columns) such that

$$
\left(B_{1}, \ldots, B_{\rho}, B_{\rho+1}\right)=(0, \ldots, 0, G) . t .
$$

The common left factor $G$ can be cancelled from (22): in effect, then, we may suppose that $G$ is unity. We apply the transformation $t$ on the left to the first $\rho+1$ rows of $M$, leaving the other rows unchanged: the complete transformation of $M$ is given by a regular matrix $T^{(\mathbf{1})}$, say, and the transformed matrix

$$
M^{(1)}=T^{(1)} M
$$

has its $(\rho+1)$-th row $R_{\rho+1}^{(1)}$ identically zero.

We now observe that a relation

$$
B_{1}^{(1)} R_{1}+\ldots+B_{\rho}^{(1)} R_{\rho}+B_{\rho+2}^{(1)} R_{\rho+2}=0
$$

exists between $R_{1}, \ldots, R_{\rho}, R_{\rho+2}$ : moreover, since $R_{1}, \ldots, R_{\rho}$ are independent, $B_{\rho+2}^{(1)}$ cannot be zero. Substituting for $R_{1}, \ldots, R_{\rho}$ in terms of the first $\rho+1$ rows of $M^{(1)}$ (we recall that $t$ is regular) we get from (23) a relation between the first $\rho+2$ rows of $M^{(1)}$. From this the term in $R_{\rho+1}^{(1)}$ can obviously be omitted: we then have a dependence relation between the first $\rho$ and the $(\rho+2)$-th rows of $M^{(1)}$. We use this dependence relation to determine a regular matrix operator $t^{(2)}$ to apply to those $\rho+1$ rows of $M^{(1)}$, leaving the other rows unchanged: the complete transformation 
is given by a regular matrix $T^{(2)}$, and the transformed matrix

$$
M^{(2)}=T^{(2)} M^{(1)}
$$

has its $(\rho+1)$-th and $(\rho+2)$-th rows identically zero.

Continuing in this way we arrive finally at a regular matrix

$$
T=\ldots T^{(2)} T^{(1)}
$$

such that in $T M$ all rows below the $\rho$-th vanish identically. This result we may express by saying that a matrix of row-rank $\rho$ may be reduced to $\rho$ rows (or fewer).

Conversely suppose that $M$ may be reduced to $\sigma$ rows, i.e.

$$
M=T N,
$$

where $T$ is a regular matrix and only the first $\sigma$ rows of $N$ are different from zero. Then

$$
M=T^{\prime} N^{\prime},
$$

where $T^{\prime}$ consists of the first $\sigma$ columns of $T$ and $N^{\prime}$ consists of the first $\sigma$ rows of $N$. Now select any $\sigma+1$ rows of $M$ to form a matrix $M^{\prime}$ and let the corresponding $\sigma+1$ rows of $T^{\prime}$ form a matrix $A$ : then

$$
M^{\prime}=A N^{\prime} .
$$

This matrix $A$ we reduce as in (20). Since $A$ has only $\sigma$ columns, $r \leqslant \sigma$ and the last row of $S A C$ must vanish identically. So also therefore do the last rows of $S A$ and of $S A N^{\prime}=S M^{\prime}$. This shows that the rows of $M^{\prime}$ are dependent: thus a matrix $M$ which can be reduced to $\sigma$ rows has rowrank $\rho \leqslant \sigma$.

Putting these two results together, we see that $\rho$ is the same as the minimum number of rows to which the matrix may be reduced (by regular multiplication on the left). Hence also the row-rank is unaltered by such regular transformation on the left (as well as on the right).

In (20), therefore, $r$ is the row-rank of $A$ as well as of $S A C$ : and any such reduction of $A$ must involve the same value of $r$.

We can define the column-rank $\kappa$ of a matrix $M$ analogously: it is the maximum number of independent columns, the minimum number of columns to which $M$ may be reduced by regular transformation on the right: and it is unaltered by regular transformation on either side.

The row-rank $\rho$ and column rank $\kappa$ are equal. To see this we first reduce column 1 of a given matrix $A$ to its first element as above. Next we reduce the first row to its first element by regular transformation on the 
right (there is no need for a transposition matrix on the left in this step). In general this will replace some or all of the zero elements in the first column by non-zero elements again: but in general also it will diminish the order of the $(1,1)$ element. We then again reduce the first column to its first element, and we continue operating alternately on the rows and on the columns as long as this diminishes the order of the $(1,1)$ element. This process terminates when we find that the element is itself the H.C.R.F. (H.C.L.F.) of the first column (row). Then one more transformation on the rows (columns), which merely subtracts left (right) multiples of the first row (column) from the others, while leaving the first row (column) itself unchanged, will produce a matrix in which both the first row and the first column are reduced to the $(1,1)$ element.

Operating now on the remaining rows and columns in the same way, we finally arrive at regular matrices $S, Q$ such that

$$
S A Q=\left(\begin{array}{cccc}
F_{1} & & & \\
& F_{2} & & \\
& & \ddots & \\
& & F_{r}
\end{array}\right)
$$

where $F_{1}, \ldots, F_{r}$ are different from zero but all other elements on the right-hand side vanish. Since regular transformation alters neither $\rho$ nor $\kappa$, we see that

$$
\rho=\kappa=r,
$$

and we may speak simply of the rank of a matrix operator.

4. Reduction, Elimination and Consistency for Simultaneous Recurrence Relations in any Number of Unknowns.

We turn now to our simultaneous equations (1). The transformation (20) gives

where

$$
\left.\begin{array}{ccccc}
F_{1} v_{1}+ & \ldots & \ldots & \ldots & \ldots=Z_{1} \\
& F_{2} v_{2}+ & \ldots & \ldots & \ldots=Z_{2} \\
& & \ldots & \ldots & \ldots \\
& & F_{r} v_{r}+ & \ldots=Z_{r}
\end{array}\right\}
$$


i.e. the $v_{i}$ are a permutation of the $u_{i}$. As to this permutation we recall that permutation is only needed when the next variable to be eliminated does not in fact occur in the remaining equations. Thus without any permutation we get

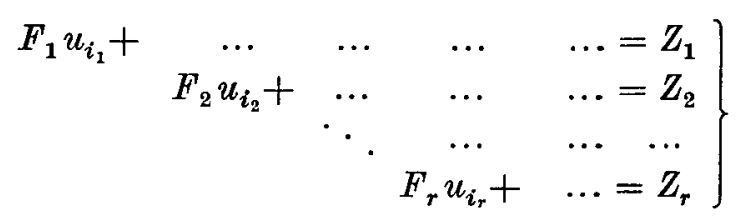

where $i_{1}<i_{2}<\ldots<i_{r}$ : the variables before $u_{i_{1}}$ do not occur, those before $u_{i_{2}}$ only occur in the first equation, and so on.

The transformation (27) gives

$$
\begin{aligned}
& F_{i} U_{i}=Z_{i} \quad(i \leqslant r), \\
& 0=Z_{i} \quad(i>r),
\end{aligned}
$$

where

$$
S z=Z, u=Q U \text { or } U=P u,
$$

$P$ being the inverse of $Q$. This transformation is less satisfactory than might at first be thought. The transformation from $u$ to $U$ lacks the geometrical interpretation of a co-ordinate transformation: $P$ and $Q$ are operator matrices. And the reduction is not unique. The result, given the initial set of equations, depends on whether we start with an H.C.R.F. or an H.C.L.F. process : and, given the procedure, two mathematically equivalent sets of equations will lead to two different reduced sets. The reduction has indeed a tendency to throw everything into the last equation rather than to separate out the difficulties. For example, one would be content with the pair of equations

$$
(E-\alpha) u=0, \quad(E-\beta) v=0 .
$$

But given the equivalent set

$$
\begin{aligned}
& (E-\alpha) u^{\prime}+(E-\alpha) v^{\prime}=0, \\
& (\beta-E) u^{\prime}+(E-\beta) v^{\prime}=0 .
\end{aligned}
$$

the H.C.R.F. process replaces this by the set

$$
\begin{gathered}
(\beta-\alpha) u^{\prime}+(2 E-\alpha-\beta) v^{\prime}=0, \\
{\left[(E-\beta)+(E-\beta) \cdot \frac{1}{\beta-\alpha} \cdot(2 E-\alpha-\beta)\right] v^{\prime}=0,}
\end{gathered}
$$


and the H.C.L.F. process then simply defines $u^{\prime \prime}$ so that the former equation becomes

$$
(\beta-\alpha) u^{\prime \prime}=0 .
$$

If we confine our transformations of variables to ordinary ones, i.e. require $P, Q$ to be matrices of numbers not of operators, then it is evident that no separation of variables is to be expected. If we allow transformation by operator matrices, the most acceptable complete separation of the system can be defined as follows. For any complete separation (32) we write down a symbol

$$
\left(n_{1}, n_{2}, \ldots, n_{r}\right),
$$

where the $n_{i}$ are the orders of the operators $F_{i}$ so arranged that

$$
n_{1} \geqslant n_{2} \geqslant \ldots \geqslant n_{r}
$$

Of two separations with symbols $\left(n_{1} \ldots\right)$ and $\left(m_{1} \ldots\right)$ we prefer the former if

$$
n_{1}<m_{1}
$$

or if

$$
n_{1}=m_{1} \text { and } n_{2}<m_{2}
$$

or if

But there is no evidence that the "most acceptable" is unique and we have no algorithm for obtaining it.

\section{The Convergents of a Continued Fraction.}

The numerator and denominator of the $n$-th convergent $r_{n}=p_{n} / q_{n}$ of the ordinary continued fraction

$$
a_{1}+\frac{1}{a_{2}+} \frac{1}{a_{3}+} \ldots
$$

satisfy

$$
\begin{gathered}
p_{n}=a_{n} p_{n-1}+p_{n-2}, \\
q_{n}=a_{n} q_{n-1}+q_{n-2} .
\end{gathered}
$$

Replacing $p_{n}$ by $q_{n} r_{n}$ we get

$$
r_{n} q_{n}=a_{n} r_{n-1} q_{n-1}+r_{n-2} q_{n-2}
$$


(35.2) and (36) can be regarded as simultaneous recurrence relations for $q_{n}$. Eliminating $q_{n}$ we get

$$
\left|\begin{array}{cccc}
1 & -a_{n+1} & --1 & 0 \\
0 & 1 & -a_{n} & -1 \\
r_{n+1} & -a_{n+1} r_{n} & -r_{n-1} & 0 \\
0 & r_{n} & -a_{n} r_{n-1} & -r_{n-2}
\end{array}\right|=0
$$

or 1

$$
a_{n} a_{n+1}\left(r_{n+1}-r_{n}\right)\left(r_{n-2}-r_{n-1}\right)=\left(r_{n+1}-r_{n-1}\right)\left(r_{n}-r_{n-2}\right) \text {, }
$$

or

$$
\left\{r_{n-2}, r_{n}, r_{n+1}, r_{n-1}\right\}=-a_{n} a_{n+1},
$$

with one of the accepted notations for a cross-ratio. Alternatively we can write

$$
\left\{r_{n-2}, r_{n-1}, r_{n}, r_{n+1}\right\}=1 /\left(1+a_{n} a_{n+1}\right)
$$

\section{The Eliminant "in general".}

We look now for a method of writing down straight away the recurrence relation for one of the variables, say $u_{k}$, and of determining the order of that relation. Here we have in mind a procedure depending only on the orders of the $A_{i j}$, not on the actual values of the coefficients $\alpha$ in those operators. For this purpose we must of course assume that the equations as given are independent and equal in number to the unknowns: thus we suppose $h=k$ and $\operatorname{rank}(A)=h$.

From what we said in $\$ 2$ it is clear that the result we obtain may fail in some way in special cases. We shall discuss this aspect partly here, but more fully in $\$ 7$.

Suppose that the $h$ equations are of orders $m_{1}, \ldots, m_{h}$ in $E$. Multiply the $i$-th equation through by

$$
1, E, E^{2}, \ldots, E^{M-m_{\iota}} \text {. }
$$

We obtain in all $\Sigma\left(M-m_{i}+1\right)$ equations. From these we wish to eliminate the quantities

$$
E^{\nu} u_{j}, \quad 0 \leqslant \nu \leqslant M, 0<j<h .
$$

The elimination is just possible if

$$
\begin{aligned}
\Sigma\left(M-m_{i}+1\right) & =(h-1)(M+1)+1, \\
M & =\Sigma m_{i} .
\end{aligned}
$$

1 From (35) one easily gets $p_{n} q_{n-1}-q_{n} p_{n-1}=(-1)^{n}, p_{n} q_{n-2}-q_{n} p_{n-2}=(-1)^{n-1} a_{n}$, well-known formulae from which (38) follows trivially. The proof given above is merely an example of the process of elimination between simultaneous recurrence relations. 
With this value of $M$, the eliminant is a determinant in which one column consists of $u_{h}$ acted on by operators, plus terms derived from the $z_{i}$, while the other columns do not involve $u_{l}$. Thus, unless the condition proves to be nugatory, we have a linear recurrence relation for $u_{h}$ : and it is in general of order $M$.

This process for the eliminant can frequently be improved, leading to a recurrence relation of lower order than (41). Suppose, for example, that the operator $A_{i j}$ is of order at most $m_{i}-\delta_{j}$. In other words, we suppose that the operators acting on $u_{j}$ are systematically of lower orders than the equation in which they occur. Then the number of quantities to be eliminated is only

$$
\underset{j<h}{\Sigma}\left(M+1-\delta_{j}\right)
$$

elimination is possible when

$$
M=\sum m_{i}-\sum_{j<h} \delta_{j}
$$

and the order of the recurrence relation for $u_{h}$ is at most

$$
M-\delta_{h}=\Sigma m_{i}-\Sigma \delta_{j} \text {. }
$$

Similarly, if the operator $A_{i j}$ has a (right) factor $E^{\epsilon_{j}}$ for all $i$, we get

$$
M=\Sigma m_{i}-\sum_{j<h}\left(\delta_{j}+\epsilon_{j}\right)
$$

and the order of the recurrence relation for $u_{h}$ is at most

$$
M-\delta_{h}-\epsilon_{h}=\Sigma m_{i}-\Sigma\left(\delta_{j}+\epsilon_{j}\right)
$$

A moment's consideration shows that if $\delta_{j}+\epsilon_{j}>0$ for some $j<h$ then the eliminant which ignores this fact is indeed nugatory: for it contains a column of zeros.

The formulae (42), (43) seem to be fairly practical. By our present methods we cannot hope to detect any lowering of the order of the recurrence relation due to relations between the coefficients $\alpha$ in the operators, and apart from such causes it is perhaps fair to say that (43) will usually give the right answer. There is, however, a better result depending only on the highest and lowest orders of $E$ in the $A_{i j}$, which we shall denote by $p_{i j}$ and $\epsilon_{i j}$ respectively. It is less easily calculable than (43).

We can get some guidance in this matter by considering the case of constant coefficients. In this case we can speak of the determinant of $A$, of the various subdeterminants, and in particular of the co-factor $A_{i j}^{\prime}$ of $A_{i j}$ in $\operatorname{det} A$. 
To eliminate $u_{1}, \ldots, u_{h-1}$, operate on the $i$-th equation with $A_{i n}^{\prime}$ and sum. A recurrence relation for $u_{h}$ alone is obtained, with $\operatorname{det} A$ as the operator. If $p$ is the highest and $\epsilon$ the lowest power of $E$ actually occurring in the expansion of $\operatorname{det} A$, we get a recurrence relation for $u_{h}$ of order

$$
p-\epsilon .
$$

From the $p_{i j}$ alone we can give an upper bound for $p$. We redefine $p$ as this bound,

$$
p=\max \Sigma p_{r, j_{\nu}},
$$

where $\left\{j_{v}\right\}$ is any permutation of the numbers $1, \ldots, h$ and the maximum is taken for all such permutations. Similarly we redefine

$$
\epsilon=\min \Sigma \epsilon_{v, j_{v}} .
$$

(44) then becames an upper bound for the order of the recurrence relation for $u_{h}$ : but it is the correct order unless the coefficients $\alpha$ obey certain relations.

The highest and lowest powers of $E$ in $A_{i n}^{\prime}$ will in general be $p_{i}{ }^{\prime}, \epsilon_{i}{ }^{\prime}$, where

$$
\begin{gathered}
p_{i}{ }^{\prime}=\max \sum_{\nu<h} p_{i_{v>},}, \\
\epsilon_{i}^{\prime}=\min \sum_{\nu<h} \epsilon_{i_{\nu,}, v}
\end{gathered}
$$

where in each case the $i_{v}$ are a permutation of the numbers $1, \ldots, h$, with $i$ itself excluded, and the maximum and minimum are taken for all such permutations.

These results for the case of constant coefficients suggest the following results, which are in fact true, for our case.

(I) If we apply the operator $E^{v}$ to the $i$-th equation for all values of $v$ from $\epsilon_{i}{ }^{\prime}$ to $p_{i}{ }^{\prime}$, we obtain in general just enough equations to eliminate the unwanted $E^{v} u_{j}(j<h)$.

(II) The order of the resulting recurrence relation for $u_{h}$ is in general $p-\epsilon$.

Before indicating the proof of these results we must refer to a point of interpretation which arises both here and in (42), (43). Suppose that a particular $A_{i j}$ vanishes identically: what values are then to be assigned 
to $p_{i j}, \epsilon_{i j}$ ? The example of operators with constant coefficients shows us that in forming our maxima and minima any combination which would involve this $p_{i j}$ or $\epsilon_{i j}$ is simply to be ignored: the same effect is obviously achieved if we write

$$
p_{i j}=-\infty, \epsilon_{i j}=+\infty \text { if } A_{i j} \equiv 0 .
$$

Any rule not equivalent to this (e.g. $\left.p_{i j}=-1, \epsilon_{i j}=+1\right)$ will on occasion lead to an "eliminant" nugatory whatever the coefficients $\alpha$.

But with this rule of interpretation some unexpected questions arise. In the argument leading to (43) we proposed to multiply the $i$-th equation through by powers of $E$ ranging from 0 to $M-m_{i}$, giving $\left(M-m_{i}+1\right)$ equations. This is nonsense unless

$$
\begin{gathered}
M-m_{i}+1 \geqslant 0, \\
\sum_{\nu \neq i} m_{v}+1 \geqslant \sum_{j<h}\left(\delta_{j}+\epsilon_{j}\right) .
\end{gathered}
$$

Even equality here would be rather alarming: it would imply that we make no use of the $i$-th equation. Similar questions arise in the more precise method leading to (44): can we be sure that $p_{i}{ }^{\prime}, \epsilon_{i}{ }^{\prime}$ as given by (46) are finite?

The solution is that (48) holds, $p_{i}{ }^{\prime}$ and $\epsilon_{i}{ }^{\prime}$ are finite, (I) and (II) are true, and the methods wo have described do work, unless the $A_{i j}$ identically zero are so distributed that there is a smaller group of, say, $h^{\prime}$ equations included in (1) which only involve $h^{\prime}-1$ unknowns other than $u_{h}$. In such a case we should obviously obtain the condition on $u_{h}$ from this more restricted set of equations.

The first step in our proof of these results is the following

LEMMA 1. Suppose that in a square block of $k^{2}$ sites, arranged in $k$ rows and $k$ columns, some sites are occupied and some unoccupied. Suppose also that there is no rectangular block, formed by the intersections of $k_{1}$ rows and $k_{2}$ columns (not necessarily consecutive), and consisting wholly of unoccupied sites, for which $k_{1}+k_{\mathbf{z}}>k$. Then there is a set of $k$ occupied sites, of which precisely one occurs in each row and precisely one in each column.

[If the "occupied sites" represent non-zero elements in a determinant, we are saying that the deterininant does not vanish identically.]

A block of unoccupied sites for which $k_{1}+k_{2}>k$ we shall call a forbidden block, one for which $k_{1}+k_{2}=k$ a maximum block of unoccupied sites. 
The proof of the lemma is, in principle, by induction on $k$.

(i) If the given array includes a maximum block ${ }^{1}$ the rest of the $k_{1}$ rows of that block form a square array of $k_{1}$ rows and $k_{1}$ columns, and we see at once that this array contains no forbidden block. So also the rest of the $k_{2}$ columns of the maximum block form a square array of $k_{2}$ rows and $k_{2}$ columns containing no forbidden block. The result follows on applying the inductive hypothesis to these smaller square arrays.

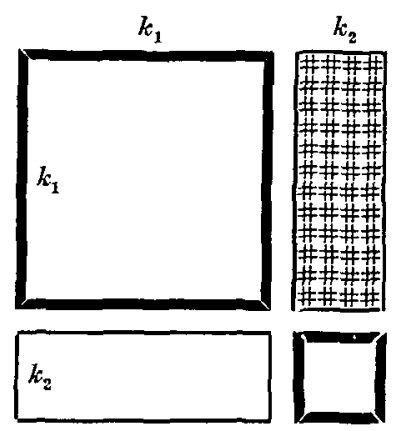

(ii) If the given square array contains no maximum block of unoccupied sites, we select an occupied site, strike out the row and column in which it lies, and find that the remaining square array of $(k-1)$ rows and $(k-1)$ columns contains no forbidden block. To it we can therefore apply the inductive hypothesis.

Since the lemma is trivial for $k=1$, its proof is now complete.

Referring to (46), (47) we see that $p_{i}{ }^{\prime}, \epsilon_{i}{ }^{\prime}$ are finite unless the submatrix of $A$ obtained by striking out the $i$-th row and last column contains a forbidden block of zeros. In this case there are $k_{1}$ equations, other than the $i$-th, which involve only $\left(k_{1}-1\right)$ variables other than $u_{h}$ : and the equation for $u_{h}$ is to be obtained from these alone.

Except in such a case, (48) follows, and with strict inequality. For there is a set $\left\{i_{v}\right\}$ for which $A_{i_{v}, \nu}$ is not zero, and we find

$$
\begin{gathered}
\epsilon_{\nu} \leqslant p_{i_{\nu \nu}} \leqslant m_{i_{\nu}}-\delta_{\nu}, \\
\sum_{\nu<h}\left(\epsilon_{\nu}+\delta_{\nu}\right) \leqslant \sum_{\nu<h} m_{i_{\nu}}=\sum_{\nu \neq i} m_{\nu} .
\end{gathered}
$$

To establish (I) we note first that the number of equations obtained is

$$
\Sigma\left(p_{i}^{\prime}-\epsilon_{i}^{\prime}+1\right) \text {. }
$$

The highest and lowest $\nu$ for which $E^{v} u_{j}$ is present in these equations are respectively

$$
p_{j}^{\prime \prime}=\max _{(i)}\left(p_{i j}+p_{i}{ }^{\prime}\right), \quad \epsilon_{j}^{\prime \prime}=\min _{(i)}\left(\epsilon_{i j}+\epsilon_{i}{ }^{\prime}\right)
$$

\footnotetext{
1 In the figure the rows and columns are rearranged so that the maximum block is in the north-east corner of the plan.
} 
and the number of variables to be eliminated is therefore

$$
\sum_{<h}\left(p_{j}^{\prime \prime}-\epsilon_{j}^{\prime \prime}+1\right) .
$$

It is therefore sufficient for us to prove that

$$
\Sigma p_{i}{ }^{\prime}=\sum_{j<h} p_{j}^{\prime \prime}, \quad \Sigma \epsilon_{i}{ }^{\prime}=\sum_{j<h} \epsilon_{j}^{\prime \prime} .
$$

We observe now that

$$
p_{j}^{\prime \prime}=\max \Sigma p_{v, j_{v}},
$$

where the maximum is taken for all sets $\left\{j_{v}\right\}$ in which the value $j$ is taken twice, all other values from 1 to $(h-1)$ taken once each. We define

$$
P=\max \Sigma p_{i_{v}, j_{v}},
$$

where the maximum is taken for all sets $\left(i_{v}, j_{v}\right)$ in which the $i_{v}$ take $(h-1)$ times each value from 1 to $h$, while the $j_{i}$ take $h$ times each value from 1 to $(h-1)$. The inequalities

$$
\Sigma p_{i}{ }^{\prime} \leqslant P, \quad \sum_{j<h} p_{j}^{\prime \prime} \leqslant P
$$

are obvious: we prove that also

$$
\Sigma p_{i}{ }^{\prime} \geqslant P, \quad \sum_{j<h} p_{j}^{\prime \prime} \geqslant P .
$$

Lemma 2. Suppose that $h(h-1)$ houses are arranged in a rectangular block of $h$ streets and $(h-1)$ avenues, one house being common to each street and each avenue. Suppose also that in each street there are $(h-1)$ occupants, and in each avenue $h$ occupants. [A particular house may be unoccupied, occupied by one person, or occupied by more than one person.] Then the occupants can be divided into $h$ parties such that the $i$-th party includes one person from each street except the $i$-th street, and one person from each avenue. And they can be dividedinto $(h-1)$ denominations, such that the $j$-th denomination includes precisely two persons from the $j$-th avenue, one person from each of the other avenues, and one person from each street.

Suppose that the first $a$ parties have been chosen, and put $h=a+b+1$. Then there remain $(b+1)$ persons in each of the first $a$ streets, $b$ persons in each of the remaining $(b+1)$ streets, and $(b+1)$ persons in each avenue. We strike out the $(a+1)$-th street and remark that the square array which is left contains no forbidden block of unoccupied houses. For if it contained an unoccupied block $k_{1} \times k_{2}$, where $k_{1}+k_{2}=h=a+b+1$, then there would be in the whole array a $k_{2} \times k_{2}$ block with $k_{2}(b+1)$ occupants. 
This is impossible since no street can have more than $(b+1)$ occupants, and one street, the $(a+1)$-th, has only $b$ occupants. Hence by Lemma 1 the $(a+1)$-th party can be chosen.

Similarly, after $a$ denominations have been chosen we form a square array from the remaining population by duplicating the $(a+1)$-th avenue, suppose that this array contains a forbidden block of empty sites, and deduce a contradiction. [There are three cases to consider, according as the supposed forbidden block involves both, one or neither of the $(a+1)$-th avenue and its duplicate.] Thus the desired result follows from Lemma 1 again.

Lemma 2 gives (54) at once, and hence, with (53), the first of the equations (50). The second of those equations is proved by the same argument: and (I) is thus established.

As for (II), it is now evident that in the last column of our determinantal eliminant occur terms $E^{\nu} u_{h}$ with values of $\nu$ ranging precisely from $\epsilon$ to $p$, so that the recurrence relation for $u_{h}$ is of order at most $(p-\epsilon)$. That it is not in general of lower order can be seen from the case of constant (but general) coefficients.

\section{Special Cases: the Nugatory Eliminant.}

We remark first that we have given more than one " general " eliminant. Each is general in a particular field of systems (1). Each such field is contained in (or contained in the closure of) a wider field : e.g., the field

$$
h=3, \quad m_{1}=m_{2}=m_{3}=5
$$

is contained in (the closure of) the field

$$
h=3, \quad m_{1}=m_{2}=m_{3}=6 .
$$

If a system (1) belonging to a field $F_{1}$ is treated as though belonging to a wider field $F_{2}$, the eliminant may go wrong in a number of ways : e.g., it may become nugatory (this occurs with the fields just cited) or give a condition necessary but not sufficient. The eliminants we have given are, in general, in the fields to which they refer, sufficient as well as necessary: but we shall not stay to establish this.

Our object in this section is to describe what can happen to a general eliminant in a special case. A thread which runs through our remarks is given by the statement that specialisation can only lower the order of the eliminant (i.e. the recurrence relation for $u_{h}$ alone). This may seem obvious : but sound reasons for it are not obvious, and indeed the statement is not entirely true. 
We have used the word field without precise definition. Such definition is possible: for our purposes a field consists effectively of systems (1) for which a particular process of elimination (a particular formula for the operators $S_{i}$, see below) gives a correct result, i.e. a condition on $u_{h}$ both necessary and sufficient.

After these preliminary remarks we observe that the eliminant is given by a row

$$
\left(S_{1}, \ldots, S_{h}\right)
$$

of operators applied to the column of equations (1). These operators are so chosen that

$$
\sum_{i} S_{i} A_{i j}=0 \quad(j<h)
$$

and the eliminant is

$$
\left(\sum_{i} S_{i} A_{i h}\right) u_{h}=\Sigma S_{i} z_{i}
$$

This is obvious in the case of $\S 4$ (and is therefore true for any field): it may be seen without difficulty in the case of the determinantal eliminants of §6. The operators $S_{i}$ have no common left factor involving $E$. This again is obvious in the case of $\S 4$, since the $S_{i}$ then form a row of a regular matrix operator: but, in any case, if $S_{i}=L S_{i}{ }^{\prime}$ then the $S_{i}{ }^{\prime}$ also satisfy (55) and hence the stronger condition

$$
\left(\Sigma S_{i}{ }^{\prime} A_{i n}\right) u_{h}=\Sigma S_{i}{ }^{\prime} z_{i}
$$

on $u_{h}$ is necessary, (56) therefore certainly not sufficient. Thus a set of operators $S_{i}$ which in general, in a field $F$, give a correct elimination have in general no common left factor: and any case in which they do have a common left factor counts as a special (or limiting) case of that field.

Finally, the set of operators giving the correct elimination is unique, apart from common left factors not involving $E$. For if there were two such, $\left(S_{1}, \ldots, S_{h}\right)$ and $\left(T_{1}, \ldots, T_{h}\right)$, these rows being independent, then the first (h-1) columns of $A$ would form a matrix operator of (row) rank at most ( $h-2)$, and so $A$ itself would be of rank less than $h$.

Now suppose that we have found a row $\left(S_{i}\right)$ of operators giving correct elimination in general in a field $F$. What then can happen to the eliminant (56) in a special or limiting case ?

The limiting ease is the limit of a sequence of systems (1) general in $F$. We correlate the members of such a sequence with the values of an integral parameter $\lambda$. The operators $S_{i}(\lambda)$ will depend on $\lambda$ (as well as on $n$ ): we ask first what happens to them as $\lambda \rightarrow \infty$. 
(I) The coefficients $\beta(\lambda)$ in the $S_{i}(\lambda)$ may fail to tend to finite limits as $\lambda \rightarrow \infty$, or they may all tend to zero.

In this case we take all the $\beta$ (the coefficients of the different powers of $E$ in the $S_{i}$ ) and regard them as homogeneous co-ordinates in a projective space, giving a point $P_{\lambda n}$ in that space. The projective space is compact, and so, for each $n$, the points $P_{\lambda n}$ have at least one limit point.

By a selection process we can replace the sequence of systems (1) by a subsequence such that, for each $n, P_{\lambda n}$ tends to a limit $P_{n}$ as $\lambda \rightarrow \infty$.

If now we normalise the operators $S_{\mathbf{i}}(\lambda)$ by the condition that the sum of the squares of the moduli of the $\beta$ be unity, the new operators

$$
S_{i}{ }^{\prime}(\lambda)=f(\lambda) \cdot S_{i}(\lambda)
$$

will converge as $\lambda \rightarrow \infty$ to operators $S_{i}^{\prime}$ which are well-defined, do not vanish identically, and satisfy (55) for the limiting case under discussion. They give an eliminant which is certainly a necessary condition, and whose order is at most equal to that of the general eliminant.

It may happen that a different sequence of systems (1) general in $F$, or a different subsequence of the first sequence, leads to a different set of operators $S_{i}^{\prime}$ for the limiting case. If so, we can treat the two eliminants as in $\$ 2$ and obtain, if they are consistent, an eliminant of lower order.

In brief, (I) may be evaded.

(II) The limiting $S_{i}$ [obtained, if necessary, by the devices of (I)] may have a common left factor. In this case we simply remove it: the resulting eliminant (57) is certainly of lower order than (56) and therefore certainly of lower order than the general eliminant.

Thus in all cases we can deduce, from operators $S_{i}(\lambda)$ giving correct elimination in the general case, a set of operators $S_{i}$ for the limiting case which satisfy (55) and have no common left factor: and the change can only lower the order of the "eliminant" so obtained.

If the system of equations (1) remains of rank $h$ in the limiting case, the condition on $u_{h}$ thus obtained is both necessary and sufficient: for we have seen that if $A$ is of rank $h$ there can be effectively only one row $\left(S_{i}\right)$ satisfying (55) and of H.C.L.F. unity.

But if the matrix operator $A$ falls in rank in the limiting case, a number of different cases may arise.

(A) The equations (1) may become inconsistent: that is, there may exist operators $T_{i}$ such that

$$
\begin{gathered}
\sum_{i} T_{i} A_{i j}=0 \quad(\text { all } j) \\
\Sigma T_{i} z_{i} \neq 0 .
\end{gathered}
$$


In this case there need by no question of the "condition on $u_{h}$ alone" or of its order. Evidently our limiting $S_{i}$ may, but will not always, reveal inconsistency.

(B) The equations (1) may remain consistent, and $u_{h}$ become arbitrary. In this case the last column of $A$ is, in the limiting case, dependent on the other columns. The limit of the eliminant (even when the $S_{i}$ have been modified as above) is nugatory. But it may be nugatory also in other cases.

(C) The equations (1) may remain consistent, and the general eliminant lead to a condition which is not nugatory. In this case the correct eliminant could be obtained as in $\$ 4$ : whatever condition on $u_{t}$ emerges, it implies our limiting eliminant and is therefore of the same or lower order.

(D) The equations (1) remain consistent, the general eliminant leads to a condition which (even when the $S_{i}$ have been modified as above) is nugatory, but $u_{h}$ is not arbitrary. In this case the true eliminant may be of higher order than in the general case. An example of this is given by the system

$$
\begin{gathered}
\alpha B_{11} u_{1}+B_{12} u_{2}=z_{1}, \\
\alpha B_{22} u_{2}=0 .
\end{gathered}
$$

Here the eliminant in the general case is $B_{22} u_{2}=0$, but in the special or limiting case $\alpha=0$ it is $B_{12} u_{2}=z_{1}$. Evidently the order of $B_{12}$ may exceed that of $B_{22}$.

\section{Generalised Continuunts.}

We turn now to the topic from which our work originated. Let $K=K_{n}$ be a determinant of $n$ rows and columns in which every element is zero except those in the principal diagonal, $k$ superdiagonals and $k$ subdiagonals. The $(i, j)$ element we may denote by

$$
\begin{gathered}
a_{i} \text { for } i=j, \\
b_{i}, c_{i}, \ldots \text { for } j-i=1,2, \ldots, k, \\
b_{j}{ }^{\prime}, c_{j}^{\prime}, \ldots \text { for } i-j=1,2, \ldots, k .
\end{gathered}
$$

For $k=1, K_{n}$ satisfies the recurrence relation

$$
K_{n}=a_{n} K_{n-1}-b_{n-1} b_{n-1}^{\prime} K_{n-2}
$$


which is of the second order and has highest coefficient unity. If there is only one subdiagonal differing from zero (and that the one next to the principal diagonal), i.e. if $c_{j}{ }^{\prime}=\ldots=0$, then $K_{n}$ satisfies a recurrence relation of order $(k+1)$, again with highest coefficient unity, which can at once be written down. For example, if $k=3$,

$K_{n}=a_{n} K_{n-1}-b_{n-1}^{\prime} b_{n-1} K_{n-2}+b_{n-1}^{\prime} b_{n-2}^{\prime} c_{n-2} K_{n-3}-b_{n-1}^{\prime} b_{n-2}^{\prime} b_{n-3}^{\prime} d_{n-3} K_{n-4}$.

Determinants of this type have been called "recurrent" by Shenton (loc. cit. \$1). In general, however, $K_{n}$ does not satisfy a recurrence relation, of fixed finite order, with highest coefficient unity, and with polynomials of fixed finite order in the elements of $K_{n}$ for its other coefficients. 'To see this we need only observe that $K_{n}$ includes terms in which we take from the columns numbered

$$
\ldots, n-5, n-3, n-1, n, n-2, n-4, \ldots
$$

respectively the elements from the rows numbered

$$
\ldots, n-3, n-1, n, n-2, n-4, n-6, \ldots,
$$

so that the completion of the cycle can be put back indefinitely.

Nevertheless, $K_{n}$ does satisfy a recurrence relation. To see this, denote by $[J]$, where

$$
J=\left(j_{1}, j_{2}, \ldots, j_{\kappa}\right)
$$

the minor obtained by striking out from $K_{n+\kappa}$ its last $\kappa$ rows and the $\kappa$ columns numbered

$$
n+\kappa-j_{1}, \ldots, n+\kappa-j_{\kappa} .
$$

We may write the symbol (59) with

$$
(0<) j_{1}<j_{2}<\ldots<j_{k},
$$

and we shall need the symbols with

$$
\kappa \leqslant k, \quad j_{k}<k+\kappa .
$$

We shall see that the functions of $n$ denoted by these symbols satisfy a set of simultaneous recurrence relations. To see first how many variables we have we remark that (59) may be replaced by

$$
\left\{j_{1}, j_{2}, \ldots, j_{k}\right\}
$$

in which, if $\kappa=k$, we have merely changed the type of bracket, while if $\kappa<k$ we complete the set of indices in (59) with the $k-\kappa$ numbers

$$
k+\kappa+1, \ldots, 2 k \text {. }
$$


The correspondence between the symbols $(59),(62)$ is easily seen to be $(1,1)$, and so the number of our variables is $\left(\begin{array}{c}2 k \\ k\end{array}\right)$. The old and new symbols for $K_{n}$ itself are

$$
\text { [( ) ], }[\{k+1, k+2, \ldots, 2 k\}] .
$$

For each of our symbols $I$ we now expand $E[I]$ by the elements of its last row. This gives a recurrence relation between our variables. This fact is obvious for $\kappa<k$, while for $\kappa=k$ we " must " take the element from the last column and last row, since all other elements of that column are zero. There are as many relations as variables, and the order of each is unity. Thus we may expect the relation for $K_{n}$ to be of order $\left(\begin{array}{c}2 k \\ k\end{array}\right)$.

We can indeed show that (44) gives the same assessment for the order of the eliminant. It is clear that $p=\left(\begin{array}{c}2 k \\ k\end{array}\right)$, and that, in a formal expansion of $\operatorname{det} A, E^{p}$ would arise only from the product of the elements in the principal diagonal. To see that $\epsilon=0$, we pick out the $(I, J)$ elements of $A$ according to the following rule:

(i) if $I=\left(i_{1}, \ldots, i_{\kappa}\right)$, where $\kappa<k$, we take $J=\left(i_{1}, \ldots, i_{\kappa}, k+\kappa\right)$,

(ii) if $I=\left(1, \ldots, m-1, i_{m}, \ldots, i_{k}\right)$, where $i_{m}>m$, we take

$$
J=\left(i_{m}-m, \ldots, i_{k}-m\right)
$$

Here we may have $m=1$, in which case the initial run $(1, \ldots, m-1)$ in $I$ is missing, or we may have $m=k+1$, in which case $i_{m}, \ldots, i_{k}$ are missing, and $J=(\quad)$.

This rule picks out one element, not involving $E$ and not in general zero, in each row of the matrix $A$. The correspondence between $I, J$ is $(1,1)$, so that $\epsilon=0$ : and actually this rule picks out the only term in the formal expansion of the determinant which does not involve $E$.

The $K_{n}$ satisfy a recurrence relation of substantially lower order if they are symmetrical. To see this we first give a different derivation for the order in the non-symmetrical case. We denote by $[I, J]$, where

$$
I, J=\left(i_{1}, \ldots, i_{\kappa} ; j_{1}, \ldots, j_{\kappa}\right),
$$

the minor obtained from $K_{n+\kappa}$ by striking out the rows numbered $n+\kappa-i_{v}$ and the columns numbered $n+\kappa-j_{v}$. We may write the symbol with

$$
i_{1}<\ldots<i_{\kappa} ; \quad j_{1}<\ldots<j_{\kappa} .
$$


We observe now that

$$
\left[\left(0, i_{2}, \ldots, i_{\kappa} ; 0, j_{2}, \ldots, j_{\kappa}\right)\right]=\left[\left(i_{2}-1, \ldots, i_{\kappa}-1 ; j_{2}-1, \ldots, j_{\kappa}-1\right)\right],
$$

a relation which we may call " reduction". We can reduce any symbol to a form in which

$$
i_{1}, j_{1} \text { do not both vanish. }
$$

We shall need the minors with

$$
i_{1}>0, \quad i_{\kappa}<k, \quad j_{\kappa}<k
$$

and also those with

$$
i_{1}=0, \quad i_{\kappa}<k, \quad j_{1}>0, \quad j_{\kappa} \leqslant k .
$$

To obtain our recurrence relations we expand $E(I, J)$ by the elements of its last row if it satisfies (A), and by the elements of its last column if it is of type (B).

The relations are all of the first order, and so the order of the eliminant is (in general) equal to the number of variables, namely

$$
\sum_{(\kappa)}\left(\begin{array}{c}
k-1 \\
\kappa
\end{array}\right) \cdot\left(\begin{array}{l}
k \\
\kappa
\end{array}\right)+\underset{(\kappa)}{\Sigma}\left(\begin{array}{l}
k-1 \\
\kappa-1
\end{array}\right) \cdot\left(\begin{array}{l}
k \\
\kappa
\end{array}\right)=\left(\begin{array}{l}
2 k \\
k
\end{array}\right)
$$

as before. When, however, $K_{n}$ is symmetrical, we have

$$
[I, J]=[J, I] \text {. }
$$

In consequence we can rule out entirely those symbols, of type (A), for which $j_{1}=0$. And of the symbols which remain under (A), we must select only one representative from each symmetrical pair $[I, J]$ and $[J, I]$, with $I \neq J$. This gives for the number of variables, and so for the order of the eliminant,

$$
\frac{1}{2} \underset{(\kappa)}{\sum}\left\{\left(\begin{array}{c}
k-1 \\
\kappa
\end{array}\right)^{2}+\left(\begin{array}{c}
k-1 \\
\kappa
\end{array}\right)\right\}+\underset{(\kappa)}{\sum}\left(\begin{array}{l}
k-1 \\
\kappa-1
\end{array}\right)\left(\begin{array}{l}
k \\
\kappa
\end{array}\right)=\frac{1}{2}\left\{\left(\begin{array}{c}
2 k-2 \\
k-1
\end{array}\right)+2^{k-1}\right\}+\left(\begin{array}{c}
2 k-1 \\
k
\end{array}\right) \text {. }
$$

Although this gives a substantial reduction, it must be admitted that the order of the eliminant increases with phenomenal rapidity with $k$, thus:

\begin{tabular}{rrr|c|c|c|c|c|c|c}
\hline$k$ & & & 0 & 1 & 2 & 3 & 4 & 5 & 6 \\
\hline Symmetrical case & $\ldots$ & $\ldots$ & 1 & 2 & 5 & 15 & 49 & 169 & 604 \\
Unsymmetrical & $\ldots$ & $\ldots$ & 1 & 2 & 6 & 20 & 70 & 252 & 924 \\
\hline
\end{tabular}

The University, Leeds. 